\title{
A Data-Driven Fault Location Algorithm Based on the Electromagnetic Time Reversal in Mismatched Media
}

\author{
Zhaoyang Wang, Member, IEEE, Zhe Chen, Student Member, IEEE, and Mario Paolone, Fellow, IEEE
}

\begin{abstract}
The classical electromagnetic time reversal (EMTR) fault location method in power systems requires multiple simulations in the backward step, assuming different guessed fault locations along the line. This process can be time consuming especially when a high location accuracy is desired, as it may require a significant number of guessed fault locations. To cope with this issue, the concept of EMTR in mismatched media has recently been introduced, allowing reducing the backward simulations to a single run and, thus, substantially improving the computation efficiency of the EMTR-based fault location technique. In this paper, we present a detailed study of the mismatched-media-based mirrored minimum energy property. This property has been applied in a few recent studies but never been theoretically studied and rigorously demonstrated. First, we infer a transfer function that relates the fault source to the voltage along the line resulting from back-injecting the time-reversed transients measured at a given observation point. We present a theorem according to which, at the fault switching frequency and its odd harmonics, the mirrorimage point of the fault location with respect to the line center corresponds to a local minimum of the squared modulus of the transfer function. Then, it is proved that the mirrored minimum energy property is a corollary of this theorem. Based on these theoretical findings, we propose an algorithm that utilizes the reversed-time voltage energy as a fault location metric in the frequency domain. We further advance a data-driven strategy to maximize the computation efficiency of the fault location procedure. The applicability and robustness of the proposed frequencydomain fault location metric are numerically and experimentally validated.
\end{abstract}

Index Terms-Data-driven methods, electromagnetic time reversal, fault location, mismatched media, power systems, transmission lines.

\section{INTRODUCTION}

C ONSIDERABLE research effort has been devoted to the problem of fault location in power networks [1]-[3]. As a result, various techniques have been proposed, which can be classified into two main categories: $i$ ) impedance- (or phasor-) based methods (e.g., [4]-[10]), and ii) traveling-wave- (or high-frequency-component-) based methods (e.g., [11]-[19]). In addition, the latest studies reflect a tendency to combine these traditional methods with artificial intelligence techniques (e.g., [20]-[24]). In the past decade, the electromagnetic time

This work is with the financial support of the Swiss Federal Office of Energy (SI/501706-01).

Z. Wang and M. Paolone are with the Electromagnetic Compatibility Laboratory and the Distributed Electrical Systems Laboratory of the Swiss Federal Institute of Technology, Lausanne (EPFL), Switzerland (e-mail: zhaoyang. wang@epfl.ch, mario.paolone@epfl.ch).

Z. Chen is with the Center for Electric Power and Energy of Technical University of Denmark (DTU), Denmark (e-mail: zhech@elektro.dtu.dk). reversal (EMTR) has been considered a promising alternative to the traditional fault location methods by taking advantage of the time-reversal invariance of wave equations (e.g., [25][29]).

The EMTR-based fault location method belongs to the traveling-wave-based category as it relies on the measurement of post-fault transient traveling waves. This category of methods is generally less sensitive to fault diversity (e.g., fault type and impedance) and less influenced by power-frequency injections of dispersed energy resources [1]. Meanwhile, in virtue of the time-reversal inherent property of refocusing time-reversed waves (e.g., fault-originated transients) to the original and unknown source, the EMTR-based method possesses a number of advantages with respect to classical traveling-wave-based methods. First, a single-end measurement suffices to locate faults as power networks behave as a closed reflective propagation medium [28]. The measurement can be carried out using either voltage transducers (e.g., resistive-capacitive voltage dividers [30]) or current probes (e.g., industrial Rogowski coils [31]) to acquire fault-induced traveling waves (either voltage or current) at the outgoing side of a substation. Secondly, it is equally applicable to power transmission and distribution systems [32]-[35]. Lastly, classical traveling-wave methods, which depend on either feature extraction or wavefront arrival time detection, generally utilize dedicated signal processing approaches, such as wavelet analysis, classification techniques as well as machine learning (e.g., [13], [22], [36]-[38]). The EMTR method applies the time-reversal operation to the full waveform of the measured transients and, if needed, the timefrequency transform (required by some of the frequency-domain metrics). In this way, the implementation of the EMTR method is characterized by a low level of complexity.

In brief, the implementation of the EMTR method is carried out through a two-step process. For the sake of illustration, let us consider a fault event along a single-wire line. Fig. 1a schematically depicts the forward propagation step (or the direct time), in which a fault occurs at $x=x_{f}$. The fault transient voltage $V_{0}^{\mathrm{DT}}(t)$ is measured at one line end (e.g., $x=0$ ). The second step, named the backward propagation step (or the reversed time), consists of numerically simulating the back-injection of the time-reversed high-frequency transients $V_{0}^{\mathrm{TR}}(t)$ from the observation point into the line, as illustrated in Fig. $1 \mathrm{~b}[28]$.

One of the fundamental hypotheses of time reversal is that the propagation media in the forward and backward steps are identical, a condition being referred to as matched media [39], 


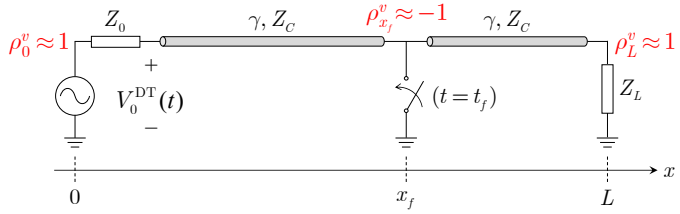

(a)

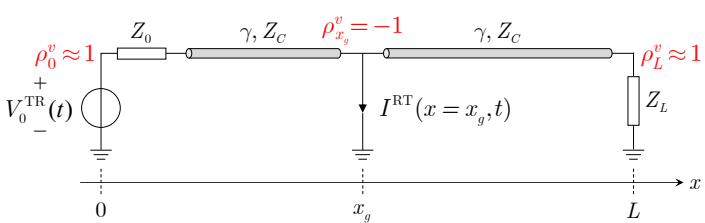

(b)

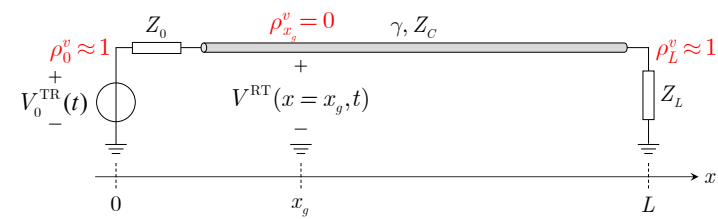

(c)

Fig. 1: Schematic representation of (a) a fault occurrence along transmission lines and the application of EMTR to locate the fault based on the conditions of: (b) matched media and (c) mismatched media. $\rho_{0}^{v}\left(\rho_{L}^{v}\right), \rho_{x_{f}}^{v}$, and $\rho_{x_{g}}^{v}$ are the voltage reflection coefficients at the line terminal $x=0(L)$, the fault location $x=x_{f}$, and the guessed fault location $x=x_{g}$, respectively. $Z_{0}\left(Z_{L}\right)$ is the input impedance of terminal power equipment.

[40]. With regard to the fault location problem, the propagation medium in the fault occurrence stage can be described by two main sets of parameters: $i$ ) the line physical characteristics, namely its length $L$, characteristic impedance $Z_{C}$ and propagation constant $\gamma$, and $i i)$ the boundary conditions at the line terminals and at the fault location.

The boundary conditions are generally described using voltage and/or current reflection coefficients [41]. Supposed being connected to power transformers, the line terminals constitute, to a first approximation, open circuits from the viewpoint of the frequency contents of fault-associated transients [42]. As a result, the terminal voltage reflection coefficient reads [41]

$$
\rho_{0}^{v}\left(\rho_{L}^{v}\right)=\frac{Z_{0}\left(Z_{L}\right)-Z_{C}}{Z_{0}\left(Z_{L}\right)+Z_{C}} \approx+1 .
$$

Conversely, the voltage reflection coefficient associated with the fault location, $\rho_{x_{f}}^{v}$, is close to -1 when a solid or low-impedance fault occurs. In the classical application of EMTR to fault location, a fault event is simulated in the backward step considering $\rho_{x_{g}}^{v}=-1$ (see Fig. 1b). In particular, the matchedmedia condition is satisfied when the guessed fault location $x_{g}$ coincides with the original fault location.

Various metrics have been proposed to quantify the time-reversed transients refocusing at the original fault location (see Table I). Razzaghi et al. advanced in 2013 the metric of fault current signal energy that portrays the true fault location with the maximum energy concentration [32]. He et al. proposed the fault current signal peak amplitude as an alternative metric to deal with situations where fault-induced transients are limited by a low signal-to-noise ratio [43]. A theoretical study was then presented to infer the correlation between the transfer
TABLE I: EMTR-based fault location metrics

\begin{tabular}{|c|c|}
\hline \multicolumn{2}{|l|}{ Matched-media-based metrics } \\
\hline Fault current signal energy (FCSE) & [32] \\
\hline Fault current signal amplitude peak ( $\infty$-norm) & [43] \\
\hline Cross-correlation sequence (MCCS) & [34], [44] \\
\hline Correlation estimator & {$[35]$} \\
\hline Root mean square & [45] \\
\hline \multicolumn{2}{|c|}{ Lumped-mismatched-media-based metrics } \\
\hline Phase angle range & [47] \\
\hline Voltage signal energy & [48], [49] \\
\hline
\end{tabular}

functions formulated in the direct time and the reversed time, respectively [35]. Taking advantage of the similarity between the back-injected time-reversed transients and the fault current signal, a metric named the maximum of cross-correlation sequences was proposed in [34], [44]. The direct-time transfer function in [34] and [35] is defined as the ratio of fault-originated transients [denoted by $V_{0}^{\mathrm{DT}}(j \omega)$ ] to fault sources. The input and output of the reversed-time transfer function are the time-reversed copy of $V_{0}^{\mathrm{DT}}(j \omega)$ and its induced fault current observed at a guessed fault location, respectively. Besides, Zhang et al. introduced the so-called mirror lines, according to which the true fault location is identified by a set of root mean square (RMS) metrics, such as the RMS value of the fault current signal that is constructed by assuming the targeted line being lossless [45].

However, $x_{f}$ being unknown before solving the fault location problem, the application of the foregoing metrics relies on first defining a set of a priori guessed fault locations (e.g., $x_{g}$ in Fig. 1b) and then simulating a fault for each of these locations. When a high location accuracy is desired, the guessed fault locations need to be defined with a high density, calling for a heavy computation burden to simulate an increasing number of backward propagation cases.

Inspired by emerging studies of time-reversal in mismatched or changing media (e.g., [46]), the concept of lumped mismatched media was recently introduced to fault location [47], [48]. To be specific, we proposed to consider a fixed topology of a non-faulty power network in the backward step, excluding the transverse branch associated with the fault, as illustrated in Fig. 1c. This would produce a mismatch between the forwardand backward-propagation media as a result of modifying the boundary condition at the guessed fault location from the state of fault (i.e., $\rho_{x_{g}}^{v}=-1$ ) to that of non-fault (i.e., $\rho_{x_{g}}^{v}=0$ ). This also constitutes a lumped mismatch in the sense that all the other line characteristics and the boundary conditions at the line extremities remain intact.

Note that such a modified backward-propagation medium does not require the independent simulation of the fault current $I^{\mathrm{RT}}(x, t)$ for each guessed fault location. More precisely, only a single simulation is needed to analyze the voltage $V^{\mathrm{RT}}$ $(x, t)$ along the non-faulty line. In this respect, its frequencydomain counterpart $V^{\mathrm{RT}}(x, j \omega)$ is proved to hold a property named bounded phase allowing an effective identification of the fault location [47].

The proposed lumped mismatched media have been numerically shown to satisfy another property named mirrored mini- 


$$
\begin{gathered}
V^{\mathrm{RT}}(x, j \omega) \triangleq V(x, j \omega)=\left(1-\rho_{0}^{v}\right) \cdot \frac{e^{-\gamma \cdot x}+\rho_{L}^{v} \cdot e^{-\gamma \cdot(2 L-x)}}{2\left[1-\rho_{0}^{v} \cdot \rho_{L}^{v} \cdot e^{-\gamma \cdot 2 L}\right]} \cdot V_{0}^{\mathrm{TR}}(j \omega) . \\
\mathcal{H}(x, j \omega)=\left(1-\rho_{0}^{v}\right) \cdot \frac{e^{-\gamma \cdot x}+\rho_{L}^{v} \cdot e^{-\gamma \cdot(2 L-x)}}{2\left[1-\rho_{0}^{v} \cdot \rho_{L}^{v} \cdot e^{-\gamma \cdot 2 L}\right]} \cdot\left[\left(1+\rho_{0}^{v}\right) \cdot \frac{e^{-\gamma \cdot x_{f}}}{1+\rho_{0}^{v} \cdot e^{-\gamma \cdot 2 x_{f}}}\right]^{*} \cdot(9) \\
|\mathcal{H}(x, j \omega)|^{2}=\overbrace{\left|1+\rho_{L}^{v} \cdot e^{-\alpha \cdot 2(L-x)} \cdot e^{-j \beta \cdot 2(L-x)}\right|^{2} \cdot e^{-\alpha \cdot 2 x}}^{\left|\mathcal{H}_{d}(x, j \omega)\right|^{2}} \frac{\left[1-\left(\rho_{0}^{v}\right)^{2}\right]^{2} \cdot e^{-\alpha \cdot 2 x_{f}}}{4\left|1-\rho_{0}^{v} \cdot \rho_{L}^{v} \cdot e^{-\alpha \cdot 2 L} \cdot e^{-j \beta \cdot 2 L}\right|^{2} \cdot\left|1+\rho_{0}^{v} \cdot e^{-\alpha \cdot 2 x_{f}} \cdot e^{j \beta \cdot 2 x_{f}}\right|^{2}} \cdot
\end{gathered}
$$

mum energy, according to which the energy of $V^{\mathrm{RT}}(x, t)$ is minimum at the mirror-image point of the fault location with respect to the line center [48]. Recently, An et al. suggested an extended algorithm applying the property with a particular focus on high-impedance fault location [49]. So far, the property itself has not been analyzed nor rigorously demonstrated. The main goal of this paper is to bridge this theoretical gap.

A thorough analysis and demonstration of this property are presented in Section II. Next, the frequency-domain calculation of the energy of $V^{\mathrm{RT}}(x, j \omega)$ as a fault location metric is proposed in Section III. The main peculiarity of the frequencydomain operation consists in collecting an array of normalized reversed-time transfer functions over a set of guessed fault locations and a range of frequencies of interest. Applying the concept of mismatched media to fault location allows reducing the simulation to a single run in the backward step. We demonstrate that it is possible to further improve the computational efficiency of the fault location procedure by leveraging a datadriven strategy, thereby advancing EMTR-based fault location towards a real-time implementation. In this regard, an acceleration algorithm is proposed in Section III. The validity and robustness of the frequency-domain metric, together with the computational efficiency of the proposed algorithm, are numerically studied in Sections III. The performance of the proposed metric is further compared in Section IV with respect to three representative traveling-wave-based metrics. Then, Section V reports an experimental validation of the proposed metric using a reduced-scale set-up. We present in Section VI a discussion about the present study, and Section VII concludes this paper with final remarks.

\section{Properties of the Direct-ReVERSED-Time TRANSFER FUNCTION}

As illustrated in Fig. 1, the fault location method of EMTR in lumped mismatched media is based on illustrating the respective propagation characteristics in the direct time and the reversed time. In this section, we analytically express the two stages' propagation characteristics in the frequency domain by formulating corresponding transfer functions. Specifically, the direct-time transfer function relates the fault event (i.e., the initial excitation $V_{f}(j \omega)$ [32], [34]) and its originated transients $V_{0}^{\mathrm{DT}}(j \omega)$. The reversed-time transfer function is the ratio of the voltage response $V^{\mathrm{RT}}(x, j \omega)$ to $V_{0}^{\mathrm{DT}}(j \omega)$ being time reversed and back injected as the secondary excitation. The direct-reversed-time transfer function is defined to combine the two stages' transfer functions and constitutes a measure to the focusing property in the mismatched-media condition.

$i$ ) The voltage observed in the direct time at the line end $x=0$ in response to a fault occurrence at $x=x_{f}$ :

$$
V_{0}^{\mathrm{DT}}(j \omega) \triangleq V(x=0, j \omega)=\frac{\left(1+\rho_{0}^{v}\right) \cdot e^{-\gamma \cdot x_{f}}}{1+\rho_{0}^{v} \cdot e^{-\gamma \cdot 2 x_{f}}} \cdot V_{f}(j \omega) \text {. }
$$

ii) The complex conjugate of $V_{0}^{\mathrm{DT}}(j \omega)$ corresponds to the time-reversed function $V_{0}^{\mathrm{DT}}(-t)$ :

$$
V_{0}^{\mathrm{DT}}(j \omega) \stackrel{\mathrm{TR}}{\longmapsto} V_{0}^{\mathrm{TR}}(j \omega): V_{0}^{\mathrm{TR}}(j \omega)=\left[V_{0}^{\mathrm{DT}}(j \omega)\right]^{*} .
$$

iii) The voltage along the line resulting from the injection of (3) at the line left end (see Fig. 1c) in the reversed time, reading $V^{\mathrm{RT}}(x, j \omega)$ in (4).

Next, to relate the above-formulated quantities, we first define the direct-time transfer function as the ratio of $V_{0}^{\mathrm{DT}}(j \omega)$ to $V_{f}(j \omega)$ according to (2), namely

$$
\mathcal{H}^{\mathrm{DT}}(j \omega)=V_{0}^{\mathrm{DT}}(j \omega) / V_{f}(j \omega)
$$

We define in a similar way the reversed-time transfer function

$$
\mathcal{H}^{\mathrm{RT}}(x, j \omega)=V^{\mathrm{RT}}(x, j \omega) / V_{0}^{\mathrm{TR}}(j \omega) .
$$

It is important to underline that $V_{0}^{\mathrm{DT}}(j \omega)$, which is obtained in the direct time, is time reversed and then behaves as an excitation in the reversed time. As a result, we can write

$$
V^{\mathrm{RT}}(x, j \omega)=\mathcal{H}^{\mathrm{RT}}(x, j \omega) \cdot\left[\mathcal{H}^{\mathrm{DT}}(j \omega)\right]^{*} \cdot\left[V_{f}(j \omega)\right]^{*} .
$$

Finally, we define the direct-reversed-time transfer function as

$$
\mathcal{H}(x, j \omega)=\mathcal{H}^{\mathrm{RT}}(x, j \omega) \cdot\left[\mathcal{H}^{\mathrm{DT}}(j \omega)\right]^{*},
$$

since it relates the output in the reversed time to the direct-time input. From (4) to $(8), \mathcal{H}(x, j \omega)$ can be analytically expressed as (9).

This way, the general task of locating faults along a transmission line can be accomplished by investigating the behavior of the direct-reversed-time transfer function only.

\section{A. Theorem: mirrored minimum squared modulus of $\mathcal{H}(x, j \omega)$}

The direct-reversed-time transfer function based on EMTR in lumped mismatched media presents a characteristics stated by the theorem as follows:

$$
\begin{aligned}
& \text { Theorem 1: } \\
& \forall f_{h} \in \mathbb{F}^{\mathrm{DT}}, \underset{0<x<L}{\operatorname{Min}}\left\{\left.|\mathcal{H}(x, j \omega)|^{2}\right|_{\omega=2 \pi \cdot f_{h}}\right\}=L-x_{f},
\end{aligned}
$$




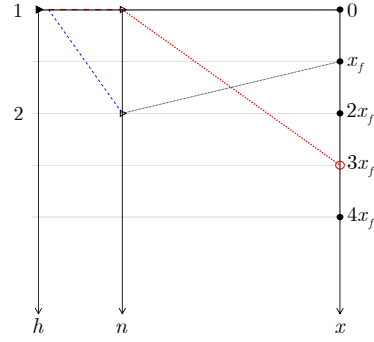

(a)

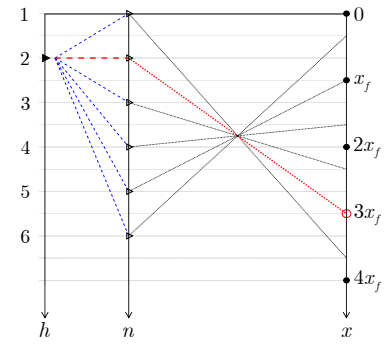

(b)

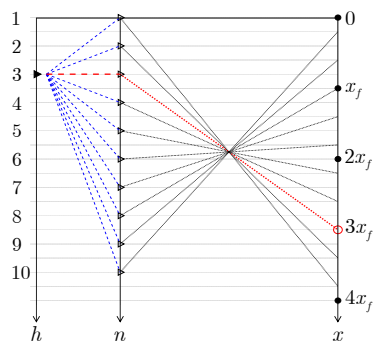

(c)

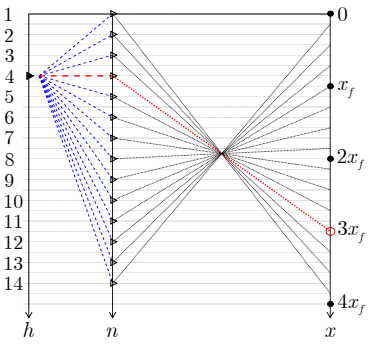

(d)

Fig. 2: Mapping among $h, n$ and $x$ of the fault case $x_{f} / L=1 / 4$ for: (a) $h=1, f=f^{0}, N_{n}=2$; (b) $h=2, f=3 f^{0}, N_{n}=6$; (c) $h=3, f=5 f^{0}, N_{n}=10$, and (d) $h=4, f=7 f^{0}, N_{n}=14$.

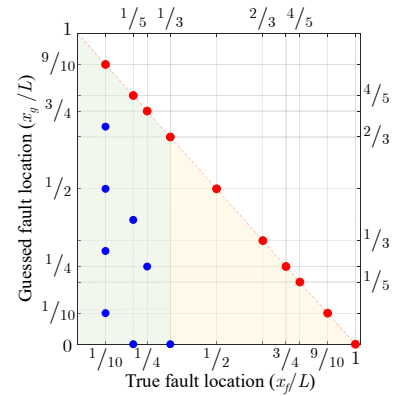

(a)

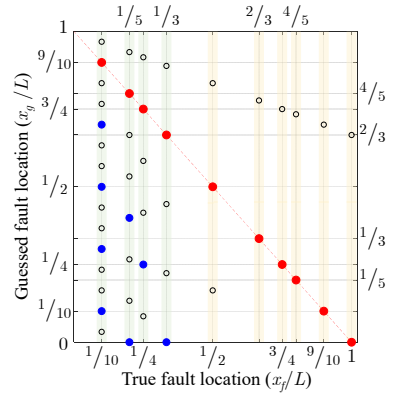

(b)

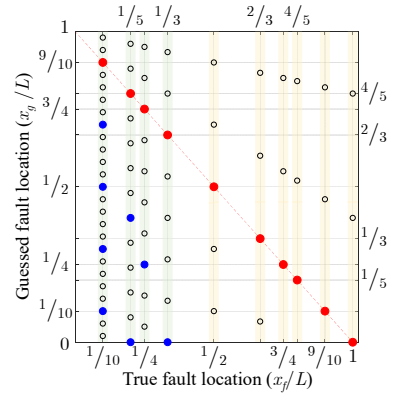

(c)

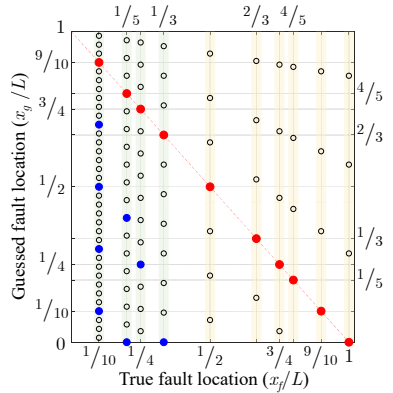

(d)

Fig. 3: Distribution of the local minima of $\left|\mathcal{H}_{d, h}(x)\right|^{2}$ for the fault cases of (18) at (a) $h=1, f=f^{0}$; (b) $h=2, f=3 f^{0}$; (c) $h=3$, $f=5 f^{0}$, and (d) $h=4, f=7 f^{0}$. Among the dots, which represent the local minima, the red dots are the mirror-image points of the respective true fault locations. In addition, for the fault case where $x_{f} \in(0, L / 3]$ the blue dots further indicate the locations of (20) that feature the local minimum of $\left|\mathcal{H}_{d, h}(x)\right|^{2}$ for each $f_{h}$.

where

$$
\begin{aligned}
\mathbb{F}^{\mathrm{DT}}=\left\{f_{h} \mid\right. & f_{h}=(2 h-1) \cdot f^{0}\left(x_{f}\right), \\
& \left.f^{0}\left(x_{f}\right)=1 /\left(4 \Gamma_{x_{f}}\right), h=1,2,3, \ldots\right\}
\end{aligned}
$$

contains the fault inherent switching frequency $f^{0}\left(x_{f}\right)$ and its odd harmonics. $\Gamma_{x_{f}}$ is the line one-way time delay of fault-originated traveling waves [34]. Unless otherwise emphasized, in what follows $f^{0}\left(x_{f}\right)$ will be concisely expressed as $f^{0}$.

Theorem 1 states that, at each single frequency among $\mathbb{F}^{\mathrm{DT}}$, the squared modulus of $\mathcal{H}(x, j \omega)$ reaches its minimum always at the mirror-image point (denoted by $x_{m i r}=L-x_{f}$ ) of the true fault location $x_{f}$, with respect to the line center.

Proof. The squared modulus of $\mathcal{H}(x, j \omega)$ is calculated as in (11) according to the low-loss line approximation ${ }^{1}$.

Obviously, only the term $\left|\mathcal{H}_{d}(x, j \omega)\right|^{2}$ in (11) is a function of the coordinate $x$, which directs the attention of the proof to discussing the behavior of $\left|\mathcal{H}_{d}(x, j \omega)\right|^{2}$ at those frequencies of $\mathbb{F}^{\mathrm{DT}}$. There exists

$$
\begin{aligned}
\left|\mathcal{H}_{d}(x, j \omega)\right|^{2}= & e^{-\alpha \cdot 2 x}+\left(\rho_{L}^{v}\right)^{2} \cdot e^{-\alpha \cdot 2(2 L-x)}+ \\
& 2 \rho_{L}^{v} \cdot e^{-\alpha \cdot 2 L} \cdot \cos [\beta \cdot 2(L-x)],
\end{aligned}
$$

\footnotetext{
${ }^{1}$ The electromagnetic transients originated by faults in power systems are generally characterized by a frequency spectrum dominating across the frequencies ranging from some kilohertz, in which the low-loss approximation in the transmission-line theory is justified for typical overhead lines and underground cables, namely $r \ll \omega \cdot l$ and $g \ll \omega \cdot c$. Given this, the characteristic impedance $Z_{C}$ is independent of frequency and essentially the same as for a lossless line [41], meanwhile, the attenuation and phase constants respectively appear to be $\alpha \cong\left(r / Z_{C}+g \cdot Z_{C}\right) / 2$ and $\beta \cong \omega \cdot \sqrt{l \cdot c}$.
}

and, at $\forall f_{h} \in \mathbb{F}^{\mathrm{DT}},\left|\mathcal{H}_{d, h}(x)\right|^{2}$ is defined by

$$
\begin{aligned}
& \left.\left|\mathcal{H}_{d}(x, j \omega)\right|^{2}\right|_{\omega=2 \pi \cdot f_{h}}= \\
& e^{-\alpha \cdot 2 x}+\left(\rho_{L}^{v}\right)^{2} \cdot e^{-\alpha \cdot 2(2 L-x)}+ \\
& 2 \rho_{L}^{v} \cdot e^{-\alpha \cdot 2 L} \cdot \cos \left[(2 h-1) \cdot \pi \cdot(L-x) / x_{f}\right] .
\end{aligned}
$$

The local minima of $\left|\mathcal{H}_{d, h}(x)\right|^{2}$ is, to a first approximation ${ }^{2}$, determined by the cos term in (13) when

$$
\begin{gathered}
(2 h-1) \cdot \pi \cdot(L-x) / x_{f}=(2 n-1) \cdot \pi, \\
h=1,2,3, \ldots, \text { and } n=0, \pm 1, \pm 2, \pm 3, \ldots .
\end{gathered}
$$

Thus, $\left|\mathcal{H}_{d, h}(x)\right|^{2}$ reaches its local minima at

$$
x=L-(2 n-1) /(2 h-1) \cdot x_{f} .
$$

Since $0<L-x_{f}, x_{f}<L$, there exist two constraints on the values of $h$ and $n$. First,

$$
h=1,2,3, \ldots, N_{h} \text { with } N_{h}=\left\lfloor f_{\max } / f^{0}\right\rfloor,
$$

where $\lfloor\cdot\rfloor$ denotes the floor function and $f_{\max }$ refers to the maximum of the frequency of interest. And for a given $h$,

$$
\begin{gathered}
n=1,2,3, \ldots, N_{n} \\
\text { with } N_{n}=\left\lfloor\left[(2 h-1) \cdot\left(L / x_{f}\right)+1\right] / 2\right\rfloor .
\end{gathered}
$$

Without limiting $h$ to a finite number, the foregoing fact applies to $\left|\mathcal{H}_{d}(x, j \omega)\right|^{2}$ at $\mathbb{F}^{\mathrm{DT}}$, thus proving Theorem 1 .

${ }^{2}$ The extrema of $\left|\mathcal{H}_{d, h}(x)\right|^{2}$ are determined by the third term of (13) because of the constant coefficient $2 \rho_{L}^{v}$. Meanwhile, the third term approximates a periodic function of $x$ since the monotonicity of $e^{-\alpha \cdot 2 L}$ is not dominant, considering that $L$ is in the order of tens to hundreds of kilometers in power grids. 
We introduce here a case study and use lattice diagrams to illustrate (15). For example, it is assumed $x_{f} / L=1 / 4$. We consider the frequencies including $f^{0}$ and its odd harmonics up to the $7^{\text {th }}$ order in such a way that $h \leqslant\left(N_{h}=4\right)$. Figs. $2 \mathrm{a}$ to $2 \mathrm{~d}$, show the mapping, among $h, n$ and $x$, governed by (15). It can be observed that, along the $x$-axis, the number as well as the locations of the local minima of $\left|\mathcal{H}_{d, h}(x)\right|^{2}$ differ in accordance with the value of $h$. As highlighted by the red lines in each of the diagrams, the mapping between $h$ and $n$ $=h$, resulting in the mirror-image point $x=x_{m i r}=3 x_{f}$ as a particular case being the location characterized by the local minimum of $\left|\mathcal{H}_{d, h}(x)\right|^{2}$ for each of the considered frequencies.

To be more general, the distribution of the local minima of $\left|\mathcal{H}_{d, h}(x)\right|^{2}$ for an arbitrary fault occurrence is shown in Fig. 3 . The considered fault cases are characterized by

$x_{f} / L=\left\{1 / 10,{ }^{1} / 5,{ }^{1} / 4,{ }^{1} / 3,{ }^{1} / 2,{ }^{2} / 3,{ }^{3} / 4,{ }^{4} / 5,9 / 10,1\right\}$.

Figures $3 \mathrm{a}$ to $3 \mathrm{~d}$ illustrate respectively those distributions at $f^{0}$ and its odd harmonics up to the $7^{\text {th }}$ order. In agreement with Theorem 1, the red dots, which appear along the downward diagonal, demonstrate that the mirror-image point is always a local minimum of $\left|\mathcal{H}_{d, h}(x)\right|^{2}$ at each $f_{h}$ and for each $x_{f}$.

\section{B. Corollary 1: mirrored minimum energy of $\mathcal{H}(x, j \omega)$}

The transfer function constitutes the frequency-domain representation to the impulse response of a linear time invariant system. According to Parseval's theorem, we calculate the ener -gy of the impulse response of the direct-reversed-time system at $\mathbb{F}^{\mathrm{DT}}$ as follows:

$$
E\left\{\left.\mathcal{H}(x, j \omega)\right|_{\mathbb{F}^{\mathrm{DT}}}\right\} \triangleq \sum_{h=1}^{N_{h}}\left\{\left.|\mathcal{H}(x, j \omega)|^{2}\right|_{\omega=2 \pi \cdot f_{h}}\right\} .
$$

There exists

$$
\text { Corollary 1: } \underset{0<x<L}{\operatorname{Min}}\left\{E\left\{\left.\mathcal{H}(x, j \omega)\right|_{\mathbb{F}^{\mathrm{DT}}}\right\}\right\}=L-x_{f} \text {. }
$$

Proof. To prove Corollary 1, we further define the set $x_{\mathbb{M}}$ of locations at which the local minimum of $\left|\mathcal{H}_{d, h}(x)\right|^{2}$ can be obtained at each $f_{h}$. For a given fault occurrence at $x=x_{f}$, $x_{\mathbb{M}}$ reads

$$
\left\{x_{m} \mid x_{m}=\left(L-x_{f}\right)-m \cdot 2 x_{f}, m=0,1,2, \ldots, N_{m}\right\} .
$$

For $x_{f} \in(L / 3, L], x_{\mathbb{M}}$ contains the mirror-image point as a single element (see the red dots in Fig. 3) while multiple local-minimum points exist for $x_{f} \in(0, L / 3]$ (see also the additional blue dots in Fig. 3). At those locations $x_{m}$,

$$
\left|\mathcal{H}_{d, h}\left(x_{m}\right)\right|^{2}=e^{-\alpha \cdot 2 x_{m}} \cdot\left[1-\rho_{L}^{v} \cdot e^{-\alpha \cdot 2\left(L-x_{m}\right)}\right]^{2} .
$$

Moreover, it can be found that $\left|\mathcal{H}_{d, h}\left(x_{m}\right)\right|^{2}$ monotonically decreases as $x_{m}$ increases.

To sum up, Corollary 1 is deduced from the properties as follows:

i) Among those locations determined by (15) to (17), $x_{m} \in$ $x_{\mathbb{M}}$ allows $|\mathcal{H}(x, j \omega)|^{2}$ to reach its local minimum at each $f_{h}$.

ii) Among $x_{\mathbb{M}}$, the mirror-image point $x_{m}=x_{m i r}=L-x_{f}$ allows $|\mathcal{H}(x, j \omega)|^{2}$ to reach its global minimum at each $f_{h}$.

iii) At the fault switching frequency $f^{0}$, the cos term of $\left|\mathcal{H}_{d, h}(x)\right|^{2}$ in (13) reaches its last local minimum at $x_{\text {mir }}$.

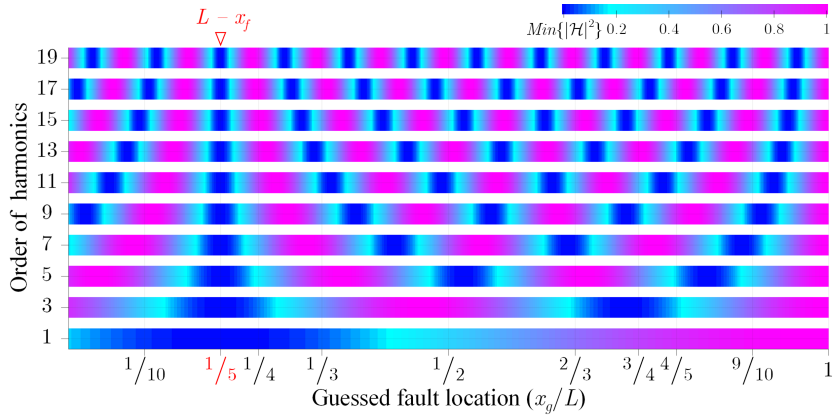

(a)

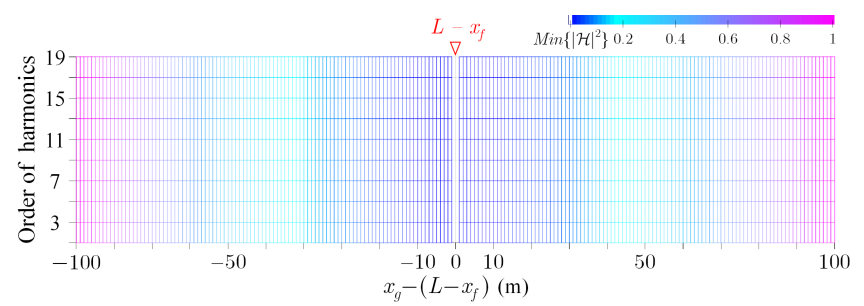

(b)

Fig. 4: Normalized squared modulus of $H\left(x_{g}, j \omega\right)$ calculated at $f^{0}$ $(=4.61 \mathrm{kHz})$ and its odd harmonics up to the $19^{\text {th }}$ order for the fault case: $L=21 \mathrm{~km}$ and $x_{f}=16.8 \mathrm{~km}$. The guessed fault locations are defined: (a) every $10 \mathrm{~m}$ along the line and (b) every $1 \mathrm{~m}$ in the spacing between $\left(L-x_{f}\right) \pm 100 \mathrm{~m}$.

The mirror-image point $x_{m i r}$ is uniquely a local minimum of $|\mathcal{H}(x, j \omega)|^{2}$ at each $f_{h} \in \mathbb{F}^{\mathrm{DT}}$, and thereby leading to the operation (19) at $x_{m i r}$, namely the summation of those minimal over $\mathbb{F}^{\mathrm{DT}}$, logically being the global minimum.

\section{Numerical validation}

In this subsection, we numerically illustrate the properties of the direct-reversed-time transfer function $\mathcal{H}(x, j \omega)$, which are stated by Theorem 1 and Corollary 1 . We consider a 21$\mathrm{km}$-long $66-\mathrm{kV}$ single-phase overhead line simulated using the EMTP-RV built-in line parameters. Various fault cases are simulated at the locations given by (18).

Exemplified by the case of $x_{f} / L=4 / 5$, we first observe in Fig. 4a the squared modulus of $\mathcal{H}(x, j \omega)$, which is distributed as a function of the guessed fault location $x_{g}$ together with the order of the harmonics $2 h-1$. For assigning the values of $x_{g}, L(=21 \mathrm{~km})$ is discretized by an increment step $\Delta x$ of $10 \mathrm{~m}$. As to $f_{h}$, we consider the maximum value of $h$ as 10 to include $f^{0}$ and its odd harmonics up to the $19^{\text {th }}$ order. For the sake of comparison, at a given harmonic $f_{h}$, the squared modulus of $\mathcal{H}(x, j \omega)$ is processed as ${ }^{3} \operatorname{Map}_{[0,1]}\left\{\left|\mathcal{H}_{h}\left(x_{g}\right)\right|^{2}\right\}$. As it can be seen, the normalized squared modulus reaches its minima at the locations satisfying the mapping relation of (15). Even though, in agreement with Theorem 1, the mirrorimage point $x_{g} / L=1 / 5$ is indicated at each harmonic by the color signifying the minimal squared modulus. Fig. $4 \mathrm{~b}$ reports a finer examination of the behavior of $\left|\mathcal{H}_{h}\left(x_{g}\right)\right|^{2}$ at the locations adjacent to the mirror-image point. To this end, the increment step $\Delta x$ is reduced to $1 \mathrm{~m}$ to define the guessed fault locations in the spacing between $\left(L-x_{f}\right) \pm 100 \mathrm{~m}$. As a result, it confirms that, on a one-meter scale, the mirror-image point 


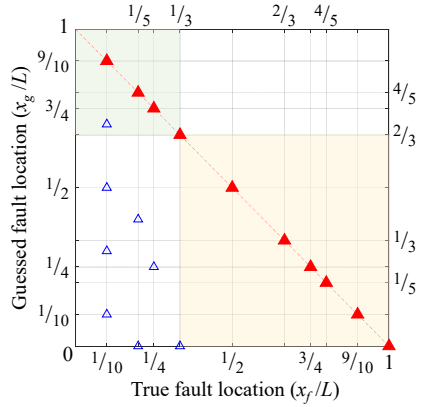

Fig. 5: Distribution of the global minima of $E\left\{\left.\mathcal{H}\left(x_{g}, j \omega\right)\right|_{F_{\mathrm{FT}}}\right\}$ (denoted by the red triangles). For the cases of $x_{f} / L \in(0,1 / 3]$, there exist additional local minimum energy points that is related to the contribution of the $f^{0}$-component of $\left|\mathcal{H}_{h}\left(x_{g}\right)\right|^{2}$. Those locations are shown by the blue triangles.

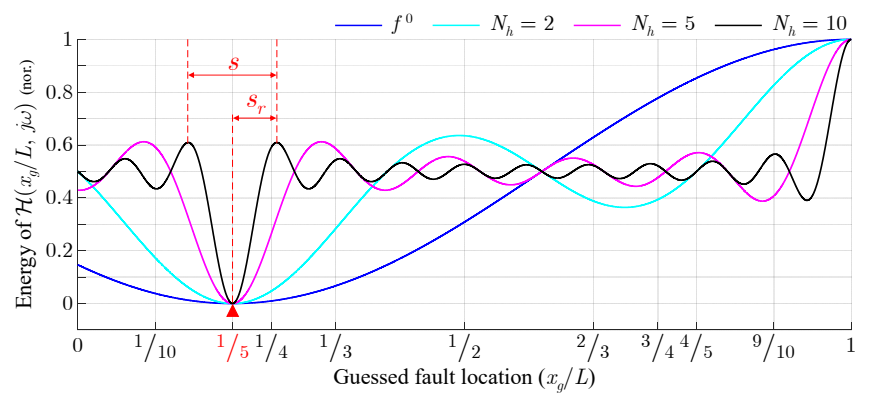

(a)

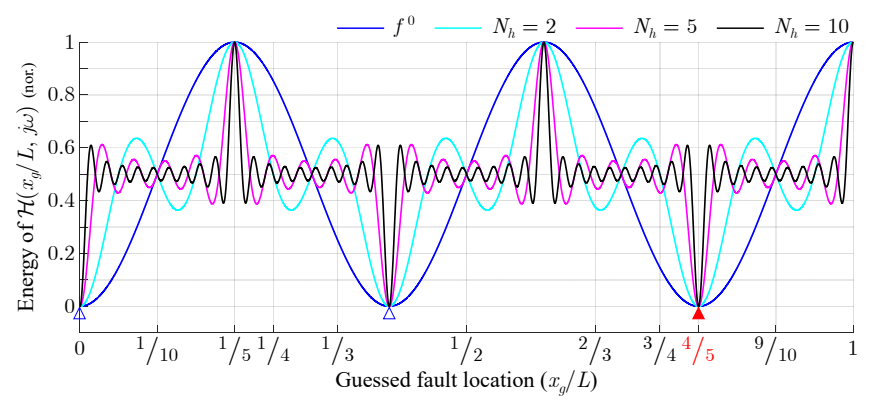

(b)

Fig. 6: Energy of $\operatorname{Map}_{[0,1]}\left\{\left|\mathcal{H}_{h}\left(x_{g}\right)\right|^{2}\right\}$ calculated at $f^{0}$ and its odd harmonics up to the $3^{\text {-rd }}, 9^{\text {th }}$, and $19^{\text {th }}$ order for the fault cases: (a) $x_{f} / L=4 / 5$ and (b) $x_{f} / L=1 / 5$. The guessed fault locations are defined by $\Delta x=1 \mathrm{~m}$. The normalization brings about that the calculated maximum energy appears to be the unit value.

uniquely features Theorem 1 .

We proceed to present in Fig. 5 the validation of the mirrored minimum energy property of $\mathcal{H}(x, j \omega)$ formulated by Corollary 1. $E\left\{\left.\mathcal{H}\left(x_{g}, j \omega\right)\right|_{\mathbb{F}^{\mathrm{DT}}}\right\}$ of (19) is calculated by considering the upper-limit frequency in $\mathbb{F}^{\mathrm{DT}}$ as the $19^{\text {th }}$ order harmonic of $f^{\circ}$. The guessed fault locations are defined with $\Delta x$ being $1 \mathrm{~m}$. The global minima of $E\left\{\left.\mathcal{H}\left(x_{g}, j \omega\right)\right|_{\mathbb{F}^{\mathrm{DT}}}\right\}$ in the respective cases are represented in Fig. 5 by red triangles. By observing that the red triangles are distributed along the downward diagonal, Corollary 1 is thus verified, indicating that the energies reach the minimum values exactly at the corresponding mirror-image points each.

To provide a detailed analysis, $\left|\mathcal{H}_{h}\left(x_{g}\right)\right|^{2}$ is normalized by

\footnotetext{
${ }^{3} \mathrm{Map}_{[0,1]}\{\mathbb{U}\}$ is a defined normalization function that maps a series of scalars $\mathbb{U}$ into a closed set $[0,1]$, showing $\operatorname{Max}\{\mathbb{U}\}=1$ and $\operatorname{Min}\{\mathbb{U}\}=0$.
}

using the function $\operatorname{Map}_{[0,1]}\{\mathbb{U}\}$. Fig. 6 depicts the curves of summating $\operatorname{Map}_{[0,1]}\left\{\left|\mathcal{H}_{h}\left(x_{g}\right)\right|^{2}\right\}$ for $f_{h}$ up to the $3^{\text {-rd }}, 9^{\text {-th }}$, and $19^{\text {th }}$ order harmonic, respectively. The fault cases $x_{f} / L=$ $4 / 5$ and $1 / 5$ are here considered as examples.

Summing up the illustrations of Figs. 5 and 6 , the following conclusions can be drawn.

i) Although the calculation adopts different numbers of the harmonics, the normalized energy of $\operatorname{Map}_{[0,1]}\left\{\left|\mathcal{H}_{h}\left(x_{g}\right)\right|^{2}\right\}$ reaches its global minimum always at the mirror-image point $x_{\text {mir }}$ in all three scenarios (see the red triangle in Fig. 6).

ii ) For the cases of $x_{f} / L \in(0,1 / 3]$, beyond $x_{m i r}$, the $f^{0}$ component of $\operatorname{Map}_{[0,1]}\left\{\left|\mathcal{H}_{h}\left(x_{g}\right)\right|^{2}\right\}$ shows additional local minima at those locations where $E\left\{\left.\mathcal{H}\left(x_{g}, j \omega\right)\right|_{\mathbb{F}^{\mathrm{DT}}}\right\}$ reaches its local minima as well (see the blue triangles in Figs. 5 and 6b).

iii) The $f^{0}$-component of $\operatorname{Map}_{[0,1]}\left\{\left|\mathcal{H}_{h}\left(x_{g}\right)\right|^{2}\right\}$ determines the location where $E\left\{\left.\mathcal{H}\left(x_{g}, j \omega\right)\right|_{F^{\mathrm{DT}}}\right\}$ shows its local or global minimum. The higher-frequency components, by comparison, contribute to an enhanced resolution in terms of distinguishing $x_{m i r}$ from its adjacent points. For instance, in the case of Fig. 6a, the focal-spot radius ${ }^{4} S_{r}$ is reduced from 6151 $\mathrm{m}$ to $1202 \mathrm{~m}$ if the upper-limit frequency in $\mathbb{F}^{\mathrm{DT}}$ is increased from $3 f^{\circ}$ to $19 f^{0}$.

\section{Fault Location Using the Mirrored Minimum ENERGy Property of THE REVERSED-Time Voltage}

Compared to the transfer function $\mathcal{H}(x, j \omega), V^{\mathrm{RT}}(x, j \omega)$ is a more direct quantity to be observed in the reversed time. This section discusses the properties of $V^{\mathrm{RT}}(x, j \omega)$ that enable identifying the true fault location.

\section{A. Corollary 2: mirrored minimum energy of $V^{\mathrm{RT}}(x, j \omega)$}

The energy of the reversed-time voltage $V^{\mathrm{RT}}(x, j \omega)$ calculated at $\mathbb{F}^{\mathrm{DT}}$ is defined as follows

$$
E\left\{\left.V^{\mathrm{RT}}(x, j \omega)\right|_{\mathbb{F}^{\mathrm{DT}}}\right\} \triangleq \sum_{h=1}^{N_{h}}\left\{\left.\left|V^{\mathrm{RT}}(x, j \omega)\right|^{2}\right|_{\omega=2 \pi \cdot f_{h}}\right\} .
$$

There exists

$$
\text { Corollary 2: } \underset{0<x<L}{\operatorname{Min}}\left\{E\left\{\left.V^{\mathrm{RT}}(x, j \omega)\right|_{\mathbb{F}^{\mathrm{DT}}}\right\}\right\}=L-x_{f} \text {. }
$$

It can be found in (2) and (4) that the excitation of $V_{f}(j \omega)$ at each $f_{h}$ is a constant (i.e., independent of the coordinate $x$ ) and, as such, it is directly inferred from Corollary 1 that $V^{\mathrm{RT}}(x, j \omega)$ equally features such a mirrored minimum energy property.

In this way, the fault location can be identified by its mirrorimage point through

$$
x_{f}=L-\operatorname{Min}_{0<x<L}\left\{E\left\{\left.V^{\mathrm{RT}}(x, j \omega)\right|_{\mathbb{F}^{\mathrm{DT}}}\right\}\right\} .
$$

\footnotetext{
${ }^{4}$ Following the approach of assessing the refocusing quality of time-reversed waves to the source point, the concept of focal spot $S$ is introduced by defining its radius $S_{r}$ as the distance between $x_{m i r}$ and the extremum point that is closest to $x_{\text {mir }}$ (see Fig. 6a).
} 


$$
E\left\{\left.V^{\mathrm{RT}}(x, j \omega)\right|_{\mathbb{F}}\right\}=\sum_{k=1}^{N_{k}}\left\{\left.\left[\left|\tilde{V}_{0}^{\mathrm{DT}}(j \omega)\right|^{2} \cdot M p_{[0,1]}\left\{\left|\mathcal{H}^{\mathrm{RT}}(x, j \omega)\right|^{2}\right\}\right]\right|_{\omega=2 \pi \cdot f_{k}}\right\} .
$$

\section{B. Proposed acceleration algorithm for calculating the energy of the reversed-time voltage}

In this subsection, we propose an efficient algorithm to calculate the reversed-time voltage energy (22) and apply the mirror-image property (23) to identify the fault location.

From a practical point of view, the fault-originated transient voltage $V_{0}^{\mathrm{DT}}(t)$ is transformed into the frequency domain as $V_{0}^{\mathrm{DT}}(j \omega)$, allowing calculating the reversed-time voltage energy (22) in the frequency domain by

$$
\sum_{h=1}^{N_{h}}\left\{\left.\left[\left|\tilde{V}_{0}^{\mathrm{DT}}(j \omega)\right|^{2} \cdot\left|\mathcal{H}^{\mathrm{RT}}(x, j \omega)\right|^{2}\right]\right|_{\omega=2 \pi \cdot f_{h}}\right\},
$$

where $\tilde{V}_{0}^{\mathrm{DT}}(j \omega)$ denotes the fault-generated high-frequency transients, resulting from filtering out low-frequency steadystate components (e.g., of $50 \mathrm{~Hz}$ ) from $V_{0}^{\mathrm{DT}}(t)$.

With the purpose of optimizing the performance of (24) in terms of fault location accuracy and computation efficiency, along with implementing the calculation of (24) through a data-driven strategy, the following considerations are in order.

i) The normalization operation $\operatorname{Map}_{[0,1]}\left\{\left|\mathcal{H}_{h}\left(x_{g}\right)\right|^{2}\right\}$ offsets the difference in magnitude between the components of $|\mathcal{H}(x, j \omega)|^{2}$ at $\mathbb{F}^{\mathrm{DT}}$, thus maximizing the effect of the higherfrequency components to enable an enhanced resolution of localizing the fault location. It is proved that the local maxima of $\left|\mathcal{H}^{\mathrm{DT}}(x, j \omega)\right|$ appearing at $\mathbb{F}^{\mathrm{DT}}$ are equal to each other [34]. The operation $\operatorname{Map}_{[0,1]}\left\{\left|\mathcal{H}_{h}\left(x_{g}\right)\right|^{2}\right\}$, therefore, can be equivalently achieved through normalizing $\left|\mathcal{H}^{\mathrm{RT}}(x, j \omega)\right|^{2}$.

ii ) Resulting from the Fourier transform, $\tilde{V}_{0}^{\mathrm{DT}}(j \omega)$ is distributed over $\mathbb{F}=\left\{0, \Delta f, \ldots, f^{\mathrm{Ny}}-\Delta f, f^{\mathrm{Ny}}\right\}$, in which $\Delta f$ is determined by the number of sampling points of $V_{0}^{\mathrm{DT}}(t)$ and the Nyquist frequency $f^{\mathrm{Ny}}$ in accordance with a given sampling rate. Corollary 2 indeed applies to the frequencies belonging to $\mathbb{F}^{\mathrm{DT}}$. Taking advantage of the fact that $\tilde{V}_{0}^{\mathrm{DT}}(j \omega)$ is dominated by its components at $\mathbb{F}^{\mathrm{DT}}$ (e.g., [34]), the voltage energy in (24) can be alternatively evaluated over $\mathbb{F}$ and, thus, avoiding a dedicated extraction of $\mathbb{F}^{\mathrm{DT}}$ from $\mathbb{F}$.

iii) As analyzed earlier, Theorem 1 and its two corollaries do not rely on the components of $\mathcal{H}^{\mathrm{RT}}(x, j \omega)$ that are present at the frequencies below the fault switching frequency $f^{0}$. Given a set of guessed fault locations $x_{\mathbb{G}}=\{0, \Delta x, \ldots, L\}$, their fault switching frequencies satisfy $f^{0}(L)<\ldots<f^{0}(\Delta x)<$ $f^{0}(0)$ [34], hence it is feasible to truncate $\mathbb{F}$ by neglecting those frequencies belonging to $\left[0, f^{0}(L)\right)$. A narrower frequency band avails a reduced computational time.

Given the above, the formula (24) calculating the energy of the reversed-time voltage can be modified as in (25), in which $\mathbb{F}$ is defined as

$$
\begin{gathered}
\mathbb{F}=\left\{f_{k} \mid f_{k}=f^{0}(L)+k \cdot \Delta f, k=0,1,2, \ldots, N_{k}\right\} \\
\text { with } N_{k}=\left\lfloor\left[f^{\text {Ny }}-f^{0}(L)\right] / \Delta f\right\rfloor .
\end{gathered}
$$

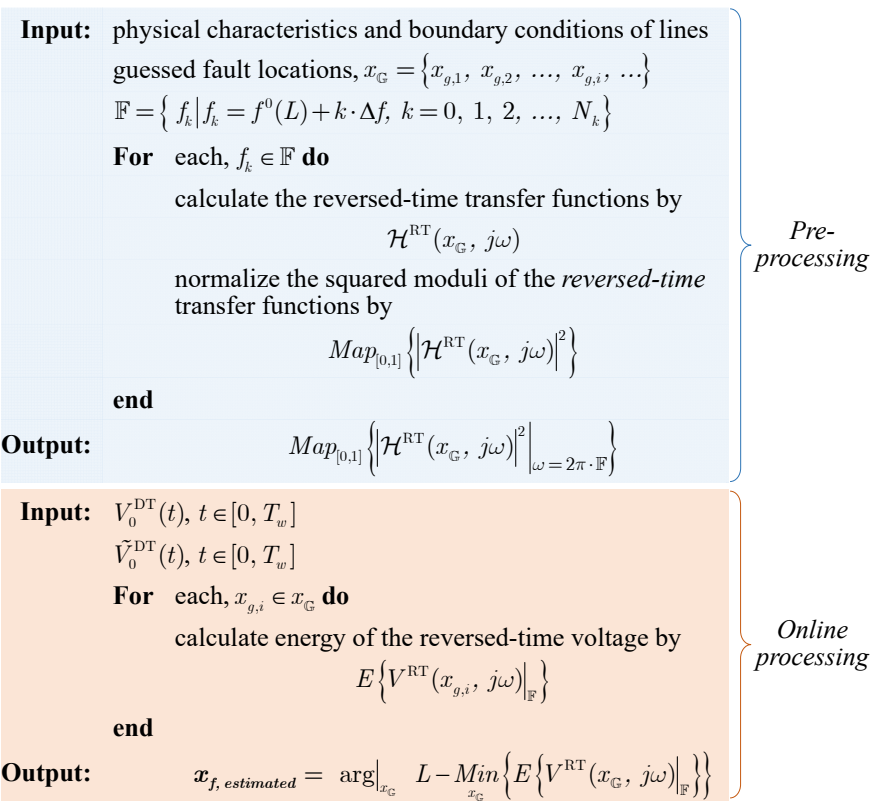

Fig. 7: Pseudo-algorithm for calculating the fault location metric of the reversed-time voltage energy.

To sum up, the step-by-step processing of computing (25) to address the fault location problem is summarized as a pseudoalgorithm presented in Fig. 7.

The algorithm starts by employing the parameters of the targeted transmission line to construct a database or dictionary, which indexes over the normalized reversed-time transfer functions $\operatorname{Map}_{[0,1]}\left\{\left|\mathcal{H}^{\mathrm{RT}}(x, j \omega)\right|^{2}\right\}$ of a set of guessed fault locations $x_{\mathbb{G}}$ and frequencies $\mathbb{F}$. As indicated as pre-processing, this procedure can be carried out prior to a fault event since it is not subject to any fault-case-dependent knowledge. Specifically, $x_{\mathbb{G}}$ is a priori assumed in line with a user-desired faultlocation accuracy. Also, in practice, $f^{N}$ and $\Delta f$ are explicit, depending on the adopted measurement capabilities (e.g., samling rates) and pre-settings (e.g., the length of the time window $T_{w}$ for recording $V_{0}^{\mathrm{DT}}(t)$ ). Thus, $\mathbb{F}$ is also known.

In the fault location stage, the traditional EMTR routine consists in back-injecting a time-reversed copy of $V_{0}^{\mathrm{DT}}(t)$ to simulate offline the backward propagation. In contrast, by employing such a data-driven strategy to pool the normalized reversed-time transfer functions beforehand, the fault location task can be accomplished quasi-instantaneously after having acquired $V_{0}^{\mathrm{DT}}(t)$.

\section{Sensitivity analysis with respect to uncertainties}

In this subsection, the proposed fault location metric of the reversed-time voltage energy (25) is examined by implementing the algorithm of Fig. 7 to cope with different fault cases, which are simulated involving the variation in $i$ ) line parameter and length, $i i$ ) fault location, and iii) fault inception, and $i v$ ) fault impedance. Moreover, the influence of noise in measurements is assessed. 
TABLE II: Fault location error of the metric (25) for the cases involving different line parameters, lengths, fault locations, and fault inception angles. The values in parentheses correspond to a fault inception angle of 2 degrees.

\begin{tabular}{|c|c|c|c|c|}
\hline & \multicolumn{4}{|c|}{ Fault location error $(\% o)$} \\
\hline$\widehat{x}_{f / L} L$ & $\begin{array}{c}21 \mathrm{~km} \\
(66 \mathrm{kV})\end{array}$ & $\begin{array}{c}45 \mathrm{~km} \\
(220 \mathrm{kV})\end{array}$ & $\begin{array}{c}75 \mathrm{~km} \\
(345 \mathrm{kV})\end{array}$ & $\begin{array}{c}120 \mathrm{~km} \\
(500 \mathrm{kV})\end{array}$ \\
\hline $1 / 10$ & $0.00(0.00)$ & 0.00 & 0.00 & $0.00(0.00)$ \\
\hline $1 / 5$ & $0.00(\overline{\mathbf{0 . 1 0}})$ & 0.00 & 0.00 & $0.00(0.00)$ \\
\hline $1 / 4$ & $0.00(0.00)$ & 0.00 & 0.00 & $0.00(0.00)$ \\
\hline $1 / 3$ & $\overline{\mathbf{0 . 0 3}}(0.03)$ & $\overline{0.03}$ & $\overline{0.03}$ & $\overline{0.03}(\overline{0.03})$ \\
\hline $1 / 2$ & $0.00(0.00)$ & 0.00 & 0.00 & $0.00(0.00)$ \\
\hline $2 / 3$ & $0.03(0.03)$ & 0.03 & 0.03 & $0.03(0.03)$ \\
\hline $3 / 4$ & $0.00(0.00)$ & 0.00 & 0.00 & $0.00(0.00)$ \\
\hline $4 / 5$ & $0.00(0.00)$ & 0.00 & 0.00 & $0.00(0.00)$ \\
\hline $9 / 10$ & $0.00(0.00)$ & 0.00 & 0.00 & $0.00(0.00)$ \\
\hline 1 & $0.00(0.00)$ & 0.00 & 0.00 & $0.00(0.00)$ \\
\hline
\end{tabular}

The simulations were carried out in the EMTP-RV environment, configuring various line set-ups with the built-in AC-66/ 220/345/500-kV constant-parameter (CP) single-phase overhead lines, and assuming a ground resistivity of $100 \Omega-\mathrm{m}$. As presented in Table II, the line length was varied from $21 \mathrm{~km}$ to $120 \mathrm{~km}$ as the rated voltage increases. For each line set-up, a single-phase-to-ground fault was simulated at the locations given by (18), one after another. A $40-\mathrm{ms}$ time window (i.e., $T_{w}=40 \mathrm{~ms}$, twice the circle duration of the $50-\mathrm{Hz}$ steadystate voltage) was used to rerecord the fault-originated transient voltage $V_{0}^{\mathrm{DT}}(t)$ at the line end, which was grounded via $100-\mathrm{k} \Omega$ impedance. The sampling rate was set at $20 \mathrm{MS} / \mathrm{s}$.

According to the above settings, the pre-processing reserves an array of the normalized reversed-time transfer functions that vary in the frequencies $\mathbb{F}$, with $\Delta f$ being $190.73 \mathrm{~Hz}$, and the guessed fault locations $x_{\mathbb{G}}$. The lower limit of $\mathbb{F}$ is determined as a function of the line length $L$. The frequencies of $f^{0}(L)$ were thus determined, according to (10), as $3.57,1.66,0.99$, and $0.62 \mathrm{kHz}$ corresponding to the considered line lengths $L$ of $21,45,75$, and $120 \mathrm{~km}$, respectively. Meanwhile, $x_{\mathbb{G}}$ was defined by specifying $\Delta x=L \times 10^{-4}$, for example, $\Delta x=2.1$ $\mathrm{m}$ for the $66-\mathrm{kV}$ line set-up.

After simulating each fault event, $V_{0}^{\mathrm{DT}}(t)$ was processed by a $4^{\text {th }}$ order Butterworth high-pass filter with a cut-off frequency of $500 \mathrm{~Hz}$, yielding the high-frequency transients $\tilde{V}_{0}^{\mathrm{DT}}(t)$. Its frequency-domain counterpart $\tilde{V}_{0}^{\mathrm{DT}}(j \omega)$, together with the built-up reversed-time transfer functions, finalized the calculation of the metric (25), providing an estimation for the mostlikely fault location.

Table II presents the fault location accuracy of the metric (25) for the considered cases involving different line parameters, lengths, as well as fault locations. The upper-bound fault location error ${ }^{5}$ in each column is indicated in bold type and with an overline. As it can be noticed, the metric (25) identifies the exact true fault location in most cases. In the few remaining cases, the errors are insignificant (a maximum of $0.03 \%$ ).

In order to evaluate the impact of the fault inception angle $\left(\theta_{f}\right)$, the simulation also involves near zero-crossing cases, in which $\theta_{f}$ is extremely small. For the sake of comparison, Table II includes the resultant error (in parentheses) in locating faults

${ }^{5}$ Fault location error $(\%)=\left|x_{f}-x_{f, \text { estimated }}\right| / L \times 1000$.
TABLE III: Fault location error of the metric (25) for the cases involving different line parameters, lengths, fault locations, and fault impedance.

\begin{tabular}{ccccccc}
\hline \multicolumn{8}{c}{ Fault location error (\%o) } \\
\hline$R_{f}(\Omega)$ & 10 & 30 & 60 & 10 & 30 & 60 \\
\hline \multicolumn{3}{c}{$L$} & \multicolumn{3}{c}{$\begin{array}{c}21 \mathrm{~km} \\
(66 \mathrm{kV})\end{array}$} & \multicolumn{4}{c}{$\begin{array}{c}120 \mathrm{~km} \\
x_{f} / L\end{array}$} & \multicolumn{5}{c}{$000 \mathrm{kV})$} \\
\hline $1 / 10$ & 0.00 & 0.00 & 0.00 & 0.00 & 0.00 & 0.00 \\
$1 / 5$ & 0.00 & 0.00 & 0.00 & 0.00 & 0.00 & 0.00 \\
$1 / 4$ & 0.00 & 0.00 & $\overline{\mathbf{0 . 1 0}}$ & 0.00 & 0.00 & 0.00 \\
$1 / 3$ & $\overline{\mathbf{0 . 0 3}}$ & $\overline{\mathbf{0 . 0 3}}$ & 0.07 & $\overline{\mathbf{0 . 0 3}}$ & $\overline{\mathbf{0 . 0 3}}$ & $\overline{\mathbf{0 . 0 3}}$ \\
$1 / 2$ & 0.00 & 0.00 & 0.00 & 0.00 & 0.00 & 0.00 \\
$2 / 3$ & 0.03 & 0.03 & 0.03 & 0.03 & 0.03 & 0.03 \\
$3 / 4$ & 0.00 & 0.00 & 0.10 & 0.00 & 0.00 & 0.00 \\
$4 / 5$ & 0.00 & 0.00 & 0.10 & 0.00 & 0.00 & 0.00 \\
$9 / 10$ & 0.00 & 0.00 & 0.10 & 0.00 & 0.00 & 0.00 \\
1 & 0.00 & 0.00 & 0.00 & 0.00 & 0.00 & 0.00 \\
\hline
\end{tabular}

TABLE IV: Fault location error of the metric (25) for the cases involving different line parameters, lengths, fault locations, and measurement noise.

\begin{tabular}{|c|c|c|c|c|c|c|}
\hline \multirow[b]{2}{*}{$S N R(\mathrm{~dB})$} & \multicolumn{6}{|c|}{ Fault location error $(\% o)$} \\
\hline & 40 & 20 & 10 & 40 & 20 & 10 \\
\hline$x_{f} / L$ & \multicolumn{3}{|c|}{$\begin{array}{l}21 \mathrm{~km} \\
(66 \mathrm{kV})\end{array}$} & \multicolumn{3}{|c|}{$\begin{array}{c}120 \mathrm{~km} \\
(500 \mathrm{kV})\end{array}$} \\
\hline $1 / 10$ & 0.00 & 0.00 & 0.40 & 0.00 & 0.00 & 0.20 \\
\hline $1 / 5$ & 0.00 & 0.20 & 0.30 & 0.00 & 0.00 & 0.10 \\
\hline $1 / 4$ & 0.00 & 0.20 & 0.20 & 0.00 & 0.00 & 0.00 \\
\hline $1 / 3$ & $\overline{0.03}$ & 0.03 & 0.13 & $\overline{0.03}$ & $\overline{0.07}$ & $\overline{0.23}$ \\
\hline $1 / 2$ & 0.00 & 0.20 & 0.10 & 0.00 & 0.00 & 0.10 \\
\hline $2 / 3$ & 0.03 & 0.07 & 0.27 & 0.03 & 0.03 & 0.07 \\
\hline $3 / 4$ & 0.00 & 0.00 & 0.50 & 0.00 & 0.00 & 0.00 \\
\hline $4 / 5$ & 0.00 & 0.10 & 0.10 & 0.00 & 0.00 & 0.10 \\
\hline $9 / 10$ & 0.00 & $\overline{0.30}$ & 0.20 & 0.00 & 0.00 & 0.10 \\
\hline 1 & 0.00 & 0.20 & $\overline{1.00}$ & 0.00 & 0.00 & 0.10 \\
\hline
\end{tabular}

with $\theta_{f}$ being 2 degrees along the 66 - and $500-\mathrm{kV}$ line. It is confirmed that the metric provides an equal-level of accuracy to locate such faults.

The performance of the metric (25) was also assessed with reference to resistive faults by assuming the fault impedance $\left(R_{f}\right)$ ranging from $10 \Omega$ to $60 \Omega$. Table III presents the fault location errors for the 66 - and $500-\mathrm{kV}$ line set-ups, considering different fault locations given by (18). It can be seen that the proposed metric features excellent location accuracy also for resistive faults.

Finally, the sensitivity of the metric (25) to noise was investigated. Table IV tabulates the fault location errors when applying Gaussian noise with different SNRs affecting the acquired voltage $V_{0}^{\mathrm{DT}}(t)$. As shown, among the results in the extreme envisaged situation with an SNR of $10 \mathrm{~dB}$, the most degraded performance appears to be a fault location error of $1 \%$, corresponding to a deviation of $21 \mathrm{~m}$.

In each of the above-mentioned cases, the metric (25) was calculated $10^{4}$ times in accordance with a precision $\Delta x$ of $L \times 10^{-4}$. With regard to the computation efficiency of the acceleration algorithm, the simulation study involves a total of 320 cases, consuming an average of 127.82-s CPU time to accomplish the online processing by means of a conventional laboratory-level computational resource: Intel E5-1620 CPU @ $3.5 \mathrm{GHz}, 16.0-\mathrm{GB}$ RAM. 


\section{Fault Location Performance Assessment of The Mirrored Minimum EnERGy Metric}

In this section, the fault location performance of the mirrored minimum energy metric is further assessed with reference to other fault location metrics belonging to the category of traveling-wave-based methods. To be specific, the following three metrics are considered:

i) The classical single-end metric [1]

$$
x_{f, \text { estimated }}=0.5 \cdot\left(t_{0}^{r}-t_{0}\right) \cdot v .
$$

ii) The classical two-end metric dependent on synchronized measurements [1]

$$
x_{f, \text { estimated }}=0.5 \cdot\left[L-\left(t_{L}-t_{0}\right) \cdot v\right] .
$$

iii) The setting-free two-end metric eliminating the need for time synchronization [15]

$$
x_{f, \text { estimated }}=\frac{\left(t_{0}^{r}-t_{0}\right)}{\left(t_{0}^{r}-t_{0}\right)+\left(t_{L}^{r}-t_{L}\right)} \cdot L .
$$

The metrics (27)-(29) require detecting the time of arrival of fault-originated traveling waves. For the sake of explanation, Fig. 8 describes the time-space distribution of the initial and reflected traveling waves caused by a fault occurrence at $x=$ $x_{f}$ along a transmission line of length $L$. The first incident traveling waves are assumed to arrive at the line ends $x=0$ and $x=L$ at the instants $t_{0}$ and $t_{L}$, respectively. $t_{0}^{r}$ and $t_{L}^{r}$ denote the respective arrival instants of the traveling waves reflected for the first time from the fault location. Besides, $v$ is the wave propagation velocity.

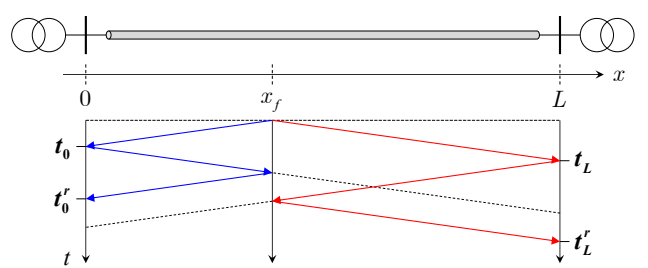

Fig. 8: Lattice-diagram illustration of fault-originated traveling waves being measured at two line terminals.

Three scenarios, which are typical in real-world applications and likely to incur fault location errors of traveling-wave-based methods, are simulated to compare the fault location performance of the proposed mirrored minimum energy metric with those of the three reference metrics.

Scenario 1: the fault-associated features are not prominent in the acquired traveling waves. For example, in the case of a slight fault inception angle and in the presence of certain fault impedance, the fault-originated traveling waves can be fast attenuated and also contaminated by background and quantization noise. This situation constitutes a common problem for the metrics under study, because it not only raises the challenge of detecting the arrival instants of the incident and reflected traveling waves but also superimposes considerable stray components onto the fault-originated transients' frequency spectrum.

In line with the above consideration, single-phase-to-ground fault events were simulated along the $120-\mathrm{km} 500-\mathrm{kV}$ line (see Section III-C) to take into account the effects of the foregoing factors: the fault was characterized by a 2-degree fault inception angle and $30-\Omega$ fault impedance; the SNR was specified as $40 \mathrm{~dB}$.

Scenario 2: in accordance with (23), (27)-(29), an inaccurate knowledge of the physical parameters $v$ and $L$ can be a source of fault location errors. In practice, prior to a transmission line being put into live operation, utilities generally conduct unenergized tests to infer the actual value of $v$. However, in spite of utilizing the Global Positioning System (GPS) to measure the overall length $L$, the manufacturing and engineering error can still exist, since the error appears to be at a relatively-small scale and, thus, is difficult to be effectively rectified. In this regard, a $1-\%$ error $\Delta L$ was introduced in the study.

Scenario 3: the imprecision in time alignment causes fault location errors for metrics, which rely on calculating the difference between the instants of fault-originated traveling waves respectively arriving at the two line ends. In the study, the fault location performance given by (28) is additionally evaluated by assuming an error $\Delta t_{L}$ in detecting $t_{L}$. By considering a typical accuracy of clock synchronization suggested by the IEEE standard [50], $\Delta t_{L}$ was set to $10 \mu \mathrm{s}$.

The continuous wavelet transform (CWT) coefficient of the fault-originated traveling waves was computed to detect the instants $t_{0}, t_{0}^{r}, t_{L}$, and $t_{L}^{r}$ [51], [52]. Daubechies4 wavelet was employed as the mother wavelet [53]. The mirrored minimum energy metric was calculated following the settings introduced in Section III-C.

Table $\mathrm{V}$ summarizes the fault location performance of the studied metrics dealing with the fault cases under the first scenario. The metrics present different magnitudes of fault location errors. As it can be seen, the classical single-end metric (27) is markedly impaired since it demands to identify both arrival instants $t_{0}$ and $t_{0}^{r}$ accurately. A minor deviation from the actual $t_{0}^{r}$ in detecting some weak reflections can result in manifest fault location errors. The setting-free metric (29) synthesizes the measurements at two line ends and mitigates, to a certain extent, the impact of erroneously recognizing $t_{0}^{r}$ or/and $t_{L}^{r}$. The classical two-end metric (28), by comparison, only requires detecting the arrival instant of the first incident traveling waves and produces improved fault location performance. The mirrored minimum energy metric (25), on the other hand, is based on the full waveform of fault-originated traveling waves without requiring the detection of the set of arrival instants, as clarified in the previous sections. It is characterized by a maximum location error of $360 \mathrm{~m}$ (i.e., $3 \%$ ).

Figure 9 presents the scatter distribution of the fault location error according to the results mentioned above. For a given fault event, the plots show the errors of the proposed metric and the reference metrics along the horizontal and vertical axes, respectively. The color-filled area in each plot indicates that the proposed metric achieves a higher level of accuracy compared to one of the reference metrics. As shown, the blue dots, which represent the first scenario, predominantly spread over the filled area, thus demonstrating the superior performance of the mirrored minimum energy metric.

The red dots in Figs. $9 \mathrm{~b}$ and $9 \mathrm{c}$ are related to the second scenario involving a 1-\% deviation from the actual line length $L$, namely $\Delta L=1.2 \mathrm{~km}$. To focus on examining the influence 
TABLE V: Fault location error of the metrics (25), (27)-(29) for the cases along the $120-\mathrm{km} 500-\mathrm{kV}$ line. The simulated fault is characterized with a 2-degree fault inception angle and $30-\Omega$ fault impedance. A 40 -dB SNR is assumed.

\begin{tabular}{|c|c|c|c|c|c|c|c|c|}
\hline & \multicolumn{2}{|c|}{ Single-end metric (27) } & \multicolumn{2}{|c|}{ Two-end metric (28) } & \multicolumn{2}{|c|}{ Setting-free metric (29) } & \multicolumn{2}{|c|}{ Proposed metric (25) } \\
\hline$x_{x_{f} / L} \quad$ Error & $\begin{array}{l}\text { Absolute } \\
\text { (m) }\end{array}$ & $\begin{array}{l}\text { Relative } \\
(\% o)\end{array}$ & $\begin{array}{l}\text { Absolute } \\
\text { (m) }\end{array}$ & $\begin{array}{l}\text { Relative } \\
(\% o)\end{array}$ & $\begin{array}{l}\text { Absolute } \\
\text { (m) }\end{array}$ & $\begin{array}{l}\text { Relative } \\
(\% o)\end{array}$ & $\begin{array}{l}\text { Absolute } \\
\text { (m) }\end{array}$ & $\begin{array}{l}\text { Relative } \\
(\%)\end{array}$ \\
\hline $1 / 10$ & 282.24 & 2.369 & 2.28 & 0.019 & 254.97 & 2.125 & 60 & 0.5 \\
\hline $1 / 5$ & 1.14 & 0.009 & 1718.05 & 14.317 & 335.53 & 2.796 & 48 & 0.4 \\
\hline $1 / 4$ & 1396.39 & 11.637 & 1396.39 & 11.637 & 1035.38 & 8.628 & 72 & 0.6 \\
\hline $1 / 3$ & 577.71 & 4.814 & 4.05 & 0.034 & 382.89 & 3.19 & 52 & 0.433 \\
\hline $1 / 2$ & 1734.18 & 14.452 & 7.49 & 0.062 & 249.79 & 2.082 & 60 & 0.5 \\
\hline $2 / 3$ & 106.13 & 0.884 & 670.5 & 5.587 & 416.98 & 3.475 & 80 & 0.667 \\
\hline $3 / 4$ & 1649.41 & 13.745 & 417.39 & 3.478 & 972.37 & 8.103 & 240 & 2 \\
\hline $4 / 5$ & 1256.21 & 10.468 & 842.22 & 7.044 & 252.98 & 2.108 & 360 & 3 \\
\hline $9 / 10$ & 1666.25 & 13.885 & 5.22 & 0.043 & 345.06 & 2.875 & 144 & 1.2 \\
\hline 1 & 2999.79 & 24.998 & 4.65 & 0.039 & - & - & 60 & 0.5 \\
\hline
\end{tabular}

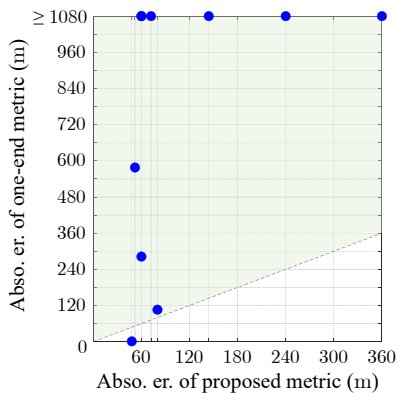

(a)

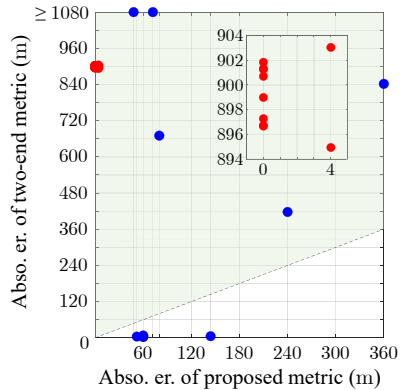

(b)

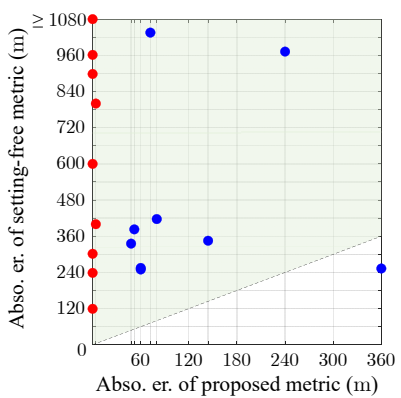

(c)

Fig. 9: Scatter distribution of the absolute fault location error. The horizontal coordinate of each dot is the absolute error of the proposed metric (25). Its vertical coordinate is the absolute error in locating the corresponding fault occurrence using one of the reference metrics: (a) (27), (b) (28), and (c) (29). The blue dot represents the scenario where the fault-associated features do not dominate the acquired traveling waves. The red dot stands for the situation in which a deviation $\Delta L$ of $1 \%$ from the actual line length $L$ exists.

of $\Delta L$, we refer to the solid fault events in Section III-C, ensuring that the set of arrival instants are correctly identified. It can be seen that the proposed metric is not affected by the considered uncertainty. In contrast, metrics (28) and (29) report the fault location errors up to $903.04 \mathrm{~m}$ (i.e., $7.525 \%$ ) and $1080.76 \mathrm{~m}$ (i.e., $9.006 \%$ o), respectively.

As analyzed previously, metric (28) enables pinpointing the fault location in some cases under the first scenario. However, compared to the other three metrics under study, its fault location performance can degrade due to incorrect time synchronization. For example, a deviation $\Delta t_{L}$ of $10 \mu \mathrm{s}$ from the actual $t_{L}$ results in the fault location errors of the ten cases with an average of $1499.28 \mathrm{~m}$ (i.e., $12.494 \%$ ).

\section{EXPERIMENTAL VALIDATION}

In this section, we present an experimental validation of the energy of the reversed-time voltage as a fault location metric by means of a reduced-scale set-up, which made use of a 477 m-long standard RG-58 cable composed of two segments of $73 \mathrm{~m}$ and $404 \mathrm{~m}$ in length, respectively. At the two ends of the set-up, the inner conductor was connected to the shield with resistors $Z_{0}=560 \Omega$ and $Z_{L}=1 \mathrm{k} \Omega$. Note that the values of the resistances are relatively high in comparison with the $50-\Omega$ characteristic impedance $Z_{C}$ of the RG- 58 cable. A DC voltage of $6 \mathrm{~V}$ supplied the set-up from the line end at $x=0$, which also served as the single observation point.

In the direct time, a solid fault was emulated by triggering a short circuit between the inner and shielding conductors of the cable. The fault was designed to occur at the junction of the two cable segments, namely $x_{f}=73 \mathrm{~m}$. For correctly producing the fault in such a reduced-scale set-up, the hardware fault emulator needs to feature a high-speed switch that is capable of changing its status in a few nanoseconds. To address this constraint, we adopted a metal-oxide semiconductor fieldeffect transistor (MOSFET) with a turn-on time of $3 \mathrm{~ns}$ [32]. The fault-induced response was measured as the voltage $V_{0}^{\mathrm{DT}}$ $(t)$ across $Z_{0}$. A 14-bit NI PCI-5122 digitizer was used to perform the measurement at a sampling rate of $100 \mathrm{MS} / \mathrm{s}$.

In the reversed time, $\tilde{V}_{0}^{\mathrm{DT}}(j \omega)$ was extracted by retaining the frequency-domain components of $V_{0}^{\mathrm{DT}}(t)$ present above $500 \mathrm{~Hz}$. The voltage energy metric (25) was calculated over the frequencies $\mathbb{F}$ ranging from $103.76 \mathrm{kHz}$ (i.e., $f^{0}(L=477)$ ) to $50 \mathrm{MHz}$. Fig. 10 depicts the curve of $E\left\{\left.V^{\mathrm{RT}}\left(x_{\mathbb{G}}, j \omega\right)\right|_{\mathbb{F}}\right\}$ as a function of the guessed fault location. $x_{\mathbb{G}}$ is defined along the cable with a discretization $\Delta x$ of $1 \mathrm{~m}$. The curve intersects with the red dash line that indicates the mirror-image point at the location $x_{g}=404 \mathrm{~m}$, which coincides exactly with the value of $L-x_{f}$ given that $L=477 \mathrm{~m}$ and $x_{f}=73 \mathrm{~m}$.

The obtained results provide an experimental evidence of the metric (25) in terms of its validity, meanwhile showing its capability of attaining a better-than-1-m fault location accuracy. Moreover, the computation efficiency of the algorithm is highlighted by a 0.94 -s consumption of the online processing. 


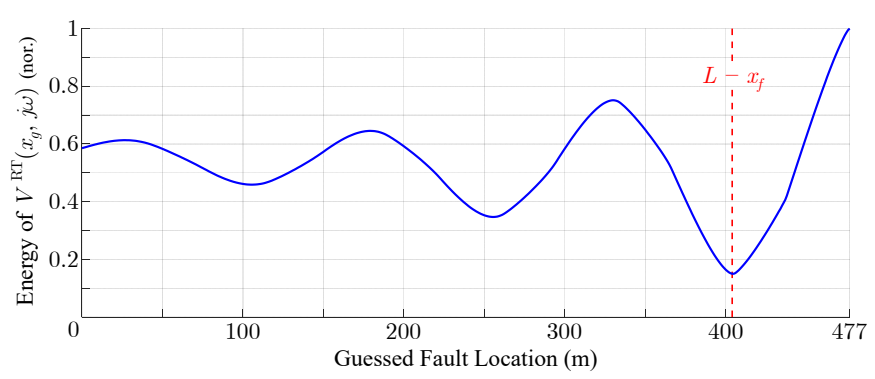

Fig. 10: Normalized energy of the reversed-time voltage as a function of the guessed fault location for the emulated fault at $x_{f}=73 \mathrm{~m}$.

\section{DISCUSSION}

The paper has provided an explicit definition, supported by a rigorous mathematical proof, of the mirrored minimum energy property of the EMTR in mismatched media. Recently, the property has been increasingly employed to develop a new branch of EMTR-based fault location methods. However, it is worth mentioning that there was a lack of rigorous investigation of the theoretical basis of this EMTR property and of the existing fault location metrics relying on it. In addition, a datadriven algorithm has been proposed, allowing the property to be utilized as a fault location metric with feasible computation efficiency and sufficient fault location accuracy.

Regarding the limitations, as clarified in Section I, EMTRbased fault location methods rely on the full waveform of fault -originated electromagnetic transients. Thus, from a practical viewpoint, the proposed metric requires measurement devices (e.g., high-voltage voltage transducers or current probes) featuring a sufficiently wide frequency bandwidth up to hundreds of $\mathrm{kHz}$ and above. Moreover, like other parameter-dependent traveling-wave methods, the fault location performance of the proposed metric is subject to inaccurate knowledge about the propagative parameters of targeted transmission lines. The line parameter errors need to be identified a priori and reduced to the largest degree through actual measurements or by taking advantage of parameter estimation techniques.

For the sake of analytically formulating the direct-reversedtime transfer function and deriving its modulus and energy characteristics versus the fault switching frequency and harmonics, the present study has considered a two-end transmission-line configuration. The existing metrics, either matched or mismatched media based (e.g., [32], [47]), have shown that EMTR fault location methods may apply to the traveling-wave propagation medium characterized by multiple scattering as a result of the branched topology of power networks. Given the above, future research should focus on extending the mirrored minimum energy metric to locate faults in complex branched power networks using single-end measurements. The presence of multi-branches causes the fault-switching-frequency component of $V_{0}^{\mathrm{DT}}(t)$ to mix with stray components, which are inherent to the complex topology of targeted transmission-line networks [11]. There are various approaches available in literature for identifying the fault-switching frequency in the frequency spectrum $V_{0}^{\mathrm{DT}}(j \omega)$. For example, the proposed metric can be coupled with the continuous-wavelet-based signal processing (i.e., [11], [54]) in view of its additional advantage of recognizing the faulty line branch.

Furthermore, optimal placement of the single observation point in complex branched power networks, with the aim of enabling the fault-switching-frequency component to dominate $V_{0}^{\mathrm{DT}}(j \omega)$, needs to be studied, given its large influence on the performance of EMTR-based fault location methods.

\section{CONCLUSIONS}

In this paper, we presented a detailed study of the mirrored minimum energy property of EMTR in lumped mismatched media, with particular reference to the fault location problem in power systems.

Firstly, we formulated a direct-reversed-time transfer function that relates the fault source signal to the voltage along the line resulting from back-injecting the time-reversed fault-generated transients. Then, we presented a theorem, according to which, at the fault switching frequency and its odd harmonics, the mirror-image point of the fault location corresponds to a local minimum of the squared modulus of the transfer function. Next, the mirrored minimum energy property is proved to be a corollary of the main theorem.

Based on these theoretical findings, we proposed an algorithm that calculates the reversed-time voltage energy as a fault location metric in the frequency domain, instead of the original time-domain approach. We demonstrated that it is possible to enhance the computational efficiency of the fault location procedure by leveraging a data-driven strategy, consisting of precalculating an array of normalized reversed-time transfer functions over a set of guessed fault locations and a range of frequencies of interest. The applicability and robustness of the frequency-domain fault location metric were numerically and experimentally validated, respectively. The proposed metric has been benchmarked against representative fault location approaches belonging to traveling-wave-based methods, demonstrating its superior performance.

Note that the proposed data-driven algorithm has been verified to be capable of assessing the fault location online quasiinstantaneously after acquiring fault-originated transients and, thus, advancing EMTR-based fault location towards a realtime implementation.

As a topic of future studies, pilot tests are expected to be conducted to experimentally validate the proposed data-driven fault location algorithm based on EMTR in mismatched media with reference to real faults in power systems. Moreover, future research needs also lie in extending the mirrored minimum energy metric to locate faults in complex branched power networks using single-end measurements.

\section{REFERENCES}

[1] M. M. Saha, J. Izykowski, and E. Rosolowski, Fault Location on Power Networks. Springer-Verlag London, 2010.

[2] M. Kezunovic, "Smart Fault Location for Smart Grids," IEEE Trans. Smart Grid, vol. 2, no. 1, pp. 11-17, Mar. 2011.

[3] IEEE Power Engineering Society, IEEE Guide for Determining Fault Location on AC Transmission and Distribution Lines, 2014.

[4] R. H. Salim, M. Resener, A. D. Filomena, K. R. C. de Oliveira, and A. S. Bretas, "Extended Fault-Location Formulation for Power Distribution Systems," IEEE Trans. Power Deliv., vol. 24, no. 2, pp. 508-516, Apr. 2009. 
[5] J. Izykowski, E. Rosolowski, P. Balcerek, M. Fulczyk, and M. M. Saha, "Accurate Noniterative Fault-Location Algorithm Utilizing TwoEnd Unsynchronized Measurements," IEEE Trans. Power Deliv., vol. 26, no. 2, pp. 547-555, Apr. 2011.

[6] Q. Jiang, B. Wang, and X. Li, "An Efficient PMU-Based Fault-Location Technique for Multiterminal Transmission Lines," IEEE Trans. Power Deliv., vol. 29, no. 4, pp. 1675-1682, Aug. 2014.

[7] T. P. S. Bains, T. S. Sidhu, Z. Xu, I. Voloh, and M. R. Zadeh, "Impedance-Based Fault Location Algorithm for Ground Faults in Series-Capacitor-Compensated Transmission Lines," IEEE Trans. Power Deliv., vol. 33, no. 1, pp. 189-199, Feb. 2018.

[8] M. Majidi and M. Etezadi-Amoli, "A New Fault Location Technique in Smart Distribution Networks Using Synchronized/Nonsynchronized Measurements," IEEE Trans. Power Deliv., vol. 33, no. 3, pp. 13581368, Jun. 2018.

[9] V. A. Stanojevi, G. Preston, S. Member, and V. Terzija, "Synchronised Measurements Based Algorithm for Long Transmission Line Fault Analysis," IEEE Trans. Smart Grid, vol. 9, no. 5, pp. 4448-4457, Sep. 2018.

[10] F. M. Aboshady, D. W. Thomas, and M. Sumner, "A New Single End Wideband Impedance Based Fault Location Scheme for Distribution Systems," Electr. Power Syst. Res., vol. 173, pp. 263-270, Aug. 2019.

[11] A. Borghetti, M. Bosetti, C. A. Nucci, M. Paolone, and A. Abur, "Integrated Use of Time-Frequency Wavelet Decompositions for Fault Location in Distribution Networks: Theory and Experimental Validation," IEEE Trans. Power Deliv., vol. 25, no. 4, pp. 3139-3146, Oct. 2010.

[12] S. Azizi, M. Sanaye-Pasand, M. Abedini, and A. Hassani, "A TravelingWave-Based Methodology for Wide-Area Fault Location in Multiterminal DC Systems," IEEE Trans. Power Deliv., vol. 29, no. 6, pp. 2552 2560, Dec. 2014.

[13] L. Ye, D. You, X. Yin, K. Wang, and J. Wu, "An Improved FaultLocation Method for Distribution System Using Wavelets and Support Vector Regression," Int. J. Electr. Power Energy Syst., vol. 55, pp. 467472, Feb. 2014.

[14] R. Liang, F. Wang, G. Fu, X. Xue, and R. Zhou, "A General Fault Location Method in Complex Power Grid Based on Wide-Area Traveling Wave Data Acquisition," Int. J. Electr. Power Energy Syst., vol. 83, pp. 213-218, Dec. 2016.

[15] F. V. Lopes, K. M. Dantas, K. M. Silva, and F. B. Costa, "Accurate TwoTerminal Transmission Line Fault Location Using Traveling Waves," IEEE Trans. Power Deliv., vol. 33, no. 2, pp. 873-880, Apr. 2018.

[16] F. V. Lopes, P. Lima, J. P. G. Ribeiro, T. R. Honorato, K. M. Silva, E. J. S. Leite, W. L. A. Neves, and G. Rocha, "Practical Methodology for Two-Terminal Traveling Wave-Based Fault Location Eliminating the Need for Line Parameters and Time Synchronization," IEEE Trans. Power Deliv., vol. 34, no. 6, pp. 2123-2134, Dec. 2019.

[17] D. Marques da Silva, F. B. Costa, V. Miranda, and H. Leite, "WaveletBased Analysis and Detection of Traveling Waves Due to DC Faults in LCC HVDC Systems," Int. J. Electr. Power Energy Syst., vol. 104, pp. 291-300, Jan. 2019

[18] O. Naidu and A. K. Pradhan, "A Traveling Wave-Based Fault Location Method Using Unsynchronized Current Measurements," IEEE Trans. Power Deliv., vol. 34, no. 2, pp. 505-513, Apr. 2019.

[19] M. Parsi, P. Crossley, P. L. Dragotti, and D. Cole, "Wavelet Based Fault Location on Power Transmission Lines Using Real-World Travelling Wave Data," Electr. Power Syst. Res., vol. 186, p. 106261, Sep. 2020.

[20] H. Livani and C. Y. Evrenosoglu, "A Fault Classification and Localization Method for Three-Terminal Circuits Using Machine Learning," IEEE Trans. Power Deliv., vol. 28, no. 4, pp. 2282-2290, Oct. 2013.

[21] H. Jiang, J. J. Zhang, W. Gao, and Z. Wu, "Fault Detection, Identification, and Location in Smart Grid Based on Data-Driven Computational Methods," IEEE Trans. Smart Grid, vol. 5, no. 6, pp. 2947-2956, Nov. 2014.

[22] A. Rafinia and J. Moshtagh, "A New Approach to Fault Location in Three-Phase Underground Distribution System Using Combination of Wavelet Analysis with ANN and FLS," Int. J. Electr. Power Energy Syst., vol. 55, pp. 261-274, Feb. 2014.

[23] Z. Jiao and R. Wu, "A New Method to Improve Fault Location Accuracy in Transmission Line Based on Fuzzy Multi-Sensor Data Fusion," IEEE Trans. Smart Grid, vol. 10, no. 4, pp. 4211-4220, Jul. 2019.

[24] S. Lan, M. Chen, and D. Chen, "A Novel HVDC Double-Terminal NonSynchronous Fault Location Method Based on Convolutional Neural Network," IEEE Trans. Power Deliv., vol. 34, no. 3, pp. 848-857, Jun. 2019.
[25] G. Lerosey, J. De Rosny, A. Tourin, A. Derode, G. Montaldo, and M. Fink, "Time Reversal of Electromagnetic Waves," Phys. Rev. Lett., vol. 92, no. 19, pp. 19-21, May 2004.

[26] J. De Rosny, G. Lerosey, and M. Fink, "Theory of Electromagnetic Time-Reversal Mirrors," IEEE Trans. Antennas Propag., vol. 58, no. 10, pp. 3139-3149, Oct. 2010.

[27] Z. Wang, F. Rachidi, M. Paolone, M. Rubinstein, and R. Razzaghi, “A Closed Time-Reversal Cavity for Electromagnetic Waves in Transmission Line Networks," IEEE Trans. Antennas Propag., vol. 69, no. 3, pp. 1621-1630, Aug. 2021.

[28] F. Rachidi, M. Rubinstein, and M. Paolone, Eds., Electromagnetic Time Reversal Application to Electromagnetic Compatibility and Power Systems. John Wiley \& Sons, 2017.

[29] Z. Wang, R. Razzaghi, M. Paolone, and F. Rachidi, "Time Reversal Applied to Fault Location in Power Networks Pilot Test: Results and Analyses," Int. J. Electr. Power Energy Syst., vol. 114, p. 105382, Jan. 2020.

[30] "Power Engineering Guide," SIEMENS, Tech. Rep., 2017.

[31] "Isolated Current and Voltage Transducers: Characteristics, Applications, Calculations," LEM, Tech. Rep., 2010.

[32] R. Razzaghi, G. Lugrin, H. Manesh, C. Romero, M. Paolone, and F. Rachidi, "An Efficient Method Based on the Electromagnetic Time Reversal to Locate Faults in Power Networks," IEEE Trans. Power Deliv., vol. 28, no. 3, pp. 1663-1673, Jul. 2013.

[33] X. Zhang, N. Tai, Y. Wang, and J. Liu, "EMTR-Based Fault Location for DC Line in VSC-MTDC System Using High-Frequency Currents," IET Gener. Transm. Distrib., vol. 11, no. 10, pp. 2499-2507, 2017.

[34] Z. Wang, R. Razzaghi, M. Paolone, and F. Rachidi, "Electromagnetic Time Reversal Similarity Characteristics and Its Application to Locating Faults in Power Networks," IEEE Trans. Power Deliv., vol. 35, no. 4, pp. 1735-1748, Aug. 2020.

[35] S. He, A. Cozza, and Y. Xie, "Electromagnetic Time Reversal as a Correlation Estimator: Improved Metrics and Design Criteria for Fault Location in Power Grids," IEEE Trans. Electromagn. Compat., vol. 62, no. 2, pp. 598-611, Jun. 2020.

[36] F. B. Costa, "Fault-Induced Transient Detection Based on Real-Time Analysis of the Wavelet Coefficient Energy," IEEE Trans. Power Deliv., vol. 29, no. 1, pp. 140-153, Feb. 2014.

[37] Y. Q. Chen, O. Fink, and G. Sansavini, "Combined Fault Location and Classification for Power Transmission Lines Fault Diagnosis With Integrated Feature Extraction," IEEE Trans. Ind. Electron., vol. 65, no. 1, pp. 561-569, Jan. 2018.

[38] L. Xie, L. Luo, Y. Li, Y. Zhang, and Y. Cao, "A Traveling WaveBased Fault Location Method Employing VMD-TEO for Distribution Network," IEEE Trans. Power Deliv., vol. 35, no. 4, pp. 1987-1998, Aug. 2020.

[39] M. Fink, "Time Reversal of Ultrasonic Fields—Part I: Basic Principles," IEEE Trans. Ultrason. Ferroelectr. Freq. Control, vol. 39, no. 5, pp. 555-566, Sep. 1992.

[40] D. Cassereau and M. Fink, "Time-Reversal of Ultrasonic Fields-Part III: Theory of the Closed Time-Reversal Cavity," IEEE Trans. Ultrason. Ferroelectr. Freq. Control, vol. 39, no. 5, pp. 579-592, Sep. 1992.

[41] C. R. Paul, Analysis of Multiconductor Transmission Lines. Wiley, 2007.

[42] A. Greenwood, Electrical Transients in Power Systems. John Wiley \& Sons, 1991.

[43] S. He, Y. Xie, Z. Wang, F. Rachidi, B. Liu, Q. Li, and X. Kong, "Norm Criteria in the Electromagnetic Time Reversal Technique for Fault Location in Transmission Lines," IEEE Trans. Electromagn. Compat., vol. 60, no. 5, pp. 1240-1248, Oct. 2018.

[44] Z. Wang, R. Razzaghi, M. Paolone, and F. Rachidi, "Electromagnetic Time Reversal Applied to Fault Location: On the Properties of BackInjected Signals," in Proc. Power Syst. Comput. Conf., 2018.

[45] X. Zhang, N. Tai, P. Wu, C. Fan, X. Zheng, and W. Huang, "A New Theory for Locating Line Fault in Power System: Theoretical Part," IEEE Access, vol. 7, pp. 91 337-91 346, Jul. 2019.

[46] D. Liu, S. Vasudevan, J. Krolik, G. Bal, and L. Carin, "Electromagnetic Time-Reversal Source Localization in Changing Media: Experiment and Analysis," IEEE Trans. Antennas Propag., vol. 55, no. 2, pp. 344-354, Feb. 2007.

[47] A. Codino, Z. Wang, R. Razzaghi, M. Paolone, and F. Rachidi, "An Alternative Method for Locating Faults in Transmission Line Networks Based on Time Reversal," IEEE Trans. Electromagn. Compat., vol. 59, no. 5, pp. 1601-1612, Mar. 2017.

[48] Z. Wang, A. Codino, R. Razzaghi, M. Paolone, and F. Rachidi, "Using Electromagnetic Time Reversal to Locate Faults in Transmission 
Lines: Definition and Application of the "Mirrored Minimum Energy" Property," in Proc. Int. Symp. Electromagn. Compat., 2017.

[49] J. An, C. Zhuang, F. Rachidi, and R. Zeng, "An Effective EMTR-Based High-Impedance Fault Location Method for Transmission Lines," IEEE Trans. Electromagn. Compat., vol. 63, no. 1, pp. 268-276, May 2021.

[50] IEEE Instrumentation and Measurement Society, IEEE Standard for a Precision Clock Synchronization Protocol for Networked Measurement and Control Systems, 2019.

[51] F. H. Magnago and A. Abur, "Fault Location Using Wavelets," IEEE Trans. Power Deliv., vol. 13, no. 4, pp. 1475-1480, Oct. 1998.

[52] C. Y. Evrenosoglu and A. Abur, "Travelling Wave Based Fault Location for Teed Circuits," IEEE Trans. Power Deliv., vol. 20, no. 2, pp. 11151121, Apr. 2005.

[53] I. Daubechies, Ten Lectures on Wavelets. Society for Industrial and Applied Mathematics, 1992.

[54] A. Borghetti, M. Bosetti, M. Di Silvestro, C. A. Nucci, and M. Paolone, "Continuous-Wavelet Transform for Fault Location in Distribution Power Networks: Definition of Mother Wavelets Inferred From Fault Originated Transients Alberto," IEEE Trans. Power Syst., vol. 23, no. 2, pp. 380-388, May 2008. 